\title{
THE HILBERT TRANSFORM WITH EXPONENTIAL WEIGHTS
}

\author{
LEONARDO COLZANI AND MARCO VIGNATI
}

(Communicated by Paul S. Muhly)

Abstract. We study the operator

$$
\mathscr{H} f(x)=2^{-x} \int_{0}^{+\infty} \frac{2^{y} f(y)}{x-y} d y
$$

on Lorentz spaces on $\mathbb{R}_{+}$with respect to the measure $4^{x} d x$. This is related to the harmonic analysis of radial functions on hyperbolic spaces. We prove that this operator is bounded on the Lorentz spaces $L^{2,9}\left(\mathbb{R}_{+}, 4^{x} d x\right), 1<q<$ $+\infty$, and it maps the Lorentz space $L^{2,1}\left(\mathbb{R}_{+}, 4^{x} d x\right)$ into a space that we call WEAK- $L^{2,1}\left(\mathbb{R}_{+}, 4^{x} d x\right)$. We also prove that $\mathscr{l}$ maps $L^{1}\left(\mathbb{R}_{+}, 4^{x} d x\right)$ into WEAK- $L^{1}\left(\mathbb{R}_{+}, 4^{x} d x\right)+L^{2}\left(\mathbb{R}_{+}, 4^{x} d x\right)$.

The radial part of the Haar measure on the three-dimensional hyperbolic space has density $(\operatorname{Sinh} 2 x)^{2} d x$, the spherical functions are $\left\{\frac{\sin \lambda x}{\lambda \operatorname{Sinh} x \operatorname{Cosh} x}\right\}$, and for suitable radial functions one has a Fourier transform and an inversion formula

$$
\begin{gathered}
\hat{f}(\lambda)=\int_{0}^{+\infty} f(y) \frac{\sin \lambda y}{\lambda \operatorname{Sinh} y \operatorname{Cosh} y}(\operatorname{Sinh} 2 y)^{2} d y, \\
f(x)=\frac{1}{2 \pi} \int_{0}^{+\infty} \hat{f}(\lambda) \frac{\sin \lambda x}{\lambda \operatorname{Sinh} x \operatorname{Cosh} x} \lambda^{2} d \lambda .
\end{gathered}
$$

(See [7].)

The $R$ th partial sums of the Fourier expansion of $f$ are defined by

$$
S_{R} f(x)=\frac{1}{2 \pi} \int_{0}^{R} \hat{f}(\lambda) \frac{\sin \lambda x}{\lambda \operatorname{Sinh} x \operatorname{Cosh} x} \lambda^{2} d \lambda,
$$

so that, by an explicit computation,

$$
\begin{aligned}
S_{R} f(x)= & \frac{1}{\pi} \int_{0}^{+\infty} \frac{\operatorname{Sinh} 2 y}{\operatorname{Sinh} 2 x} \frac{\sin R(x-y)}{x-y} f(y) d y \\
& -\frac{1}{\pi} \int_{0}^{+\infty} \frac{\operatorname{Sinh} 2 y}{\operatorname{Sinh} 2 x} \frac{\sin R(x+y)}{x+y} f(y) d y .
\end{aligned}
$$

Received by the editors July 30,1990; these results have been presented at the Conferences on Interpolation Spaces and Related Topics, Technion, Haifa, Israel, July 1990.

1980 Mathematics Subject Classification (1985 Revision). Primary 43A50, 44A15.

Key words and phrases. Hilbert transform, Lorentz spaces, hyperbolic spaces. 
This operator naturally leads to consider the Hilbert transform

$$
(\operatorname{Sinh} 2 x)^{-1} H[f(y) \operatorname{Sinh} 2 y](x)=\frac{1}{\pi \operatorname{Sinh} 2 x} \int_{0}^{+\infty} \frac{f(y) \operatorname{Sinh} 2 y}{x-y} d y
$$

on $\mathbb{R}_{+}$with the measure $(\operatorname{Sinh} 2 x)^{2} d x$.

The local behavior of this operator can be studied using the theory of the weights $A_{p}$, but, since the measure $(\operatorname{Sinh} 2 x)^{2} d x$ has an exponential growth, the $A_{p}$ theory does not apply globally. Indeed, it can easily be seen that this operator is bounded on $L^{p}\left(\mathbb{R}_{+},(\operatorname{Sinh} 2 x)^{2} d x\right)$ only if $p=2$. However more precise results can be obtained in terms of Lorentz spaces.

In this paper we study the related operator

$$
\mathscr{H} f(x)=2^{-x} \int_{0}^{+\infty} \frac{2^{y} f(y)}{x-y} d y
$$

on Lorentz spaces on $\mathbb{R}_{+}$with respect to the measure $4^{x} d x$. In particular, we prove the following result.

Theorem. (i) The operator $\mathscr{H}$ is bounded on the Lorentz spaces $L^{2, q}\left(\mathbb{R}_{+}, 4^{x} d x\right)$, $1<q<+\infty$

(ii) $\mathscr{H}$ is bounded from $L^{2,1}\left(\mathbb{R}_{+}, 4^{x} d x\right)$ into WEAK- $L^{2,1}\left(\mathbb{R}_{+}, 4^{x} d x\right)$.

(iii) $\mathscr{H}$ is bounded from $L^{1}\left(\mathbb{R}_{+}, 4^{x} d x\right)$ into WEAK- $L^{1}\left(\mathbb{R}_{+}, 4^{x} d x\right)+$ WEAK- $L^{2,1}\left(\mathbb{R}_{+}, 4^{x} d x\right)$.

The definition of the space WEAK- $L^{2,1}\left(\mathbb{R}_{+}, 4^{x} d x\right)$ is a natural generalization of the definition of the Lorentz spaces $L^{2, q}\left(\mathbb{R}_{+}, 4^{x} d x\right)$, and is given in a moment. Here we only observe that if $f$ has compact support in $\mathbb{R}_{+}$and $\int_{0}^{+\infty} 2^{y} f(y) d y=1$, then if $x \rightarrow+\infty, \mathscr{H} f(x) \simeq 2^{-x} /(1+x)$. This is not a function in $L^{2,1}\left(\mathbb{R}_{+}, 4^{x} d x\right)$, but it belongs to $\bigcap_{q>1} L^{2, q}\left(\mathbb{R}_{+}, 4^{x} d x\right)$ and it is the typical example of function in WEAK- $L^{2,1}\left(\mathbb{R}_{+}, 4^{x} d x\right)$. In particular, this example shows that the theorem is essentially sharp.

The plan of the paper is the following: the first section contains definitions and some preliminary results on Lorentz spaces, and the second section is devoted to the proof of the theorem. In the final remark we briefly consider Fourier integral expansions of radial functions on the hyperbolic space.

\section{DEFINITIONS AND PRELIMINARY RESULTS}

Definition. Let $(X, \mu)$ be a measure space. The Lorentz space $L^{p, q}(X, \mu)$, $0<p<+\infty, 0<q \leq+\infty$, is the class of measurable functions with finite quasinorm

$$
\|f\|_{L^{p, q}}= \begin{cases}\left\{q \int_{0}^{+\infty}\left[t \mu\{x \in X:|f(x)|>t\}^{1 / p}\right]^{q} d t / t\right\}^{1 / q} & \text { if } q<+\infty, \\ \operatorname{Sup}_{t>0} t \mu\{x \in X:|f(x)|>t\}^{1 / p} & \text { if } q=+\infty .\end{cases}
$$

The Lorentz space $L^{p, q, r}(X, \mu), 0<p, q<+\infty, 0<r \leq+\infty$, is defined by requiring that $t \mu\{x \in X:|f(x)|>t\}^{1 / p}$ is in $L^{q, r}\left(\mathbb{R}_{+}, d t / t\right)$.

Let $f^{* *}(t)=\operatorname{Sup}_{\mu(E)<t} t^{-1} \int_{E}|f| d \mu$. For $1<p<+\infty$, an equivalent definition of the spaces $L^{p, q}(X, \mu)$ and $L^{p, q, r}(X, \mu)$ is given by requiring that the sequences $\left\{4^{j / p} f^{* *}\left(4^{j}\right)\right\}$ belong to $l^{q}(\mathbb{Z})$ and $l^{q, r}(\mathbb{Z})$ respectively. 
We also recall that, for the real method of interpolation,

$$
\begin{aligned}
& \left(L^{p_{0}, q_{0}}(X, \mu), L^{p_{1}, q_{1}}(X, \mu)\right)_{\vartheta, q}=L^{p, q}(X, \mu) \quad \text { if } \frac{1-\vartheta}{p_{0}}+\frac{\vartheta}{p_{1}}=\frac{1}{p}, \\
& \left(L^{p, q_{0}}(X, \mu), L^{p, q_{1}}(X, \mu)\right)_{\vartheta, r}=L^{p, q, r}(X, \mu) \text { if } \frac{1-\vartheta}{q_{0}}+\frac{\vartheta}{q_{1}}=\frac{1}{q} .
\end{aligned}
$$

For the spaces $L^{p, q}(X, \mu)$ see [2, 3]; for $L^{p, q, r}(X, \mu)$ see [1].

Throughout the paper, instead of writing $L^{p, q, \infty}(X, \mu)$, we prefer to write WEAK- $L^{p, q}(X, \mu)$. Also, we mainly work with the measure space $X=\mathbb{R}_{+}$and $d \mu=4^{x} d x$, and we are particularly interested in the space WEAK- $L^{2,1}\left(\mathbb{R}_{+}, 4^{x} d x\right)$. As an example, useful in the sequel, the functions $2^{-x} /(1+|x-k|), k=0,1, \ldots$, are uniformly in WEAK- $L^{2,1}\left(\mathbb{R}_{+}, 4^{x} d x\right)$ but not in $L^{2,1}\left(\mathbb{R}_{+}, 4^{x} d x\right)$. Indeed, it is easy to check that

$$
\begin{aligned}
\left(\frac{2^{-x}}{1+|x-k|}\right)^{* *}\left(4^{j}\right) & \approx \begin{cases}1 & \text { if } j \leq 0, \\
4^{-j} \int_{0}^{j} \frac{2^{-x}}{1+|x-k|} d x & \text { if } j>0,\end{cases} \\
& \approx \begin{cases}1 & \text { if } j \leq 0, \\
\frac{2^{-j}}{1+|j-k|} & \text { if } j>0 .\end{cases}
\end{aligned}
$$

Further examples of functions in these spaces can be obtained by using the following arguments.

Definition. To every sequence $\left\{\alpha_{k}\right\}$ on $\mathbb{N}$ we associate the function $T\left\{\alpha_{k}\right\}$ on $\mathbb{R}_{+}$defined by

$$
T\left\{\alpha_{k}\right\}(x)=\alpha_{k} 2^{-x} \quad \text { if }[x]=k
$$

( $[x]$ denotes the integral part of $x$ ).

To every function $f$ locally integrable on $\mathbb{R}_{+}$we associate the sequence $T^{*} f$ on $\mathbb{N}$ defined by

$$
T^{*} f(k)=\int_{k}^{k+1} 2^{x} f(x) d x
$$

Proposition. (i) The operator $\mathscr{T}$ is bounded from $l^{q}(\mathbb{N}), 0<q \leq+\infty$, into $L^{2, q}\left(\mathbb{R}_{+}, 4^{x} d x\right)$.

(ii) $\mathscr{T}$ is bounded from WEAK- $l^{1}(\mathbb{N})$ into WEAK- $L^{2,1}\left(\mathbb{R}_{+}, 4^{x} d x\right)$.

(iii) $\mathscr{T}^{*}$ is bounded from $L^{2, q}\left(\mathbb{R}_{+}, 4^{x} d x\right), 1 \leq q \leq+\infty$, into $l^{q}(\mathbb{N})$.

Proof. Proof of (i). Since $\left\|2^{-x} \chi_{[k, k+1)}(x)\right\|_{L^{2, q}} \leq c$, with $c$ independent of $k=0,1, \ldots$, the result holds if $0<q \leq 1$. Since the function $2^{-x}$ is in $L^{2, \infty}\left(\mathbb{R}_{+}, 4^{x} d x\right)$, the result holds if $q=+\infty$. By interpolation we obtain the result if $1<q<+\infty$.

Proof of (ii). Consider the sublinear operator

$$
S\left\{\alpha_{k}\right\}\left(4^{j}\right)=2^{j}\left[T\left\{\alpha_{k}\right\}\right]^{* *}\left(4^{j}\right) .
$$

By (i) $S$ is bounded from $l^{q}(\mathbb{N}), 0<q \leq+\infty$, into $l^{q}(\mathbb{Z})$. Hence by interpolation, $S$ is bounded from $l^{1, \infty}(\mathbb{N})$ into WEAK- $l^{1}(\mathbb{Z})$. 

$T$.

Proof of (iii). This follows from (i) and the fact that $T^{*}$ is the adjoint of

\section{PROOF OF THE THEOREM}

Proof of (i) and (ii). Let

and decompose

$$
\begin{cases}\varphi_{k}= \begin{cases}\chi_{[0,2)} & \text { if } k=0, \\ \chi_{[k-1, k+2)} & \text { if } k=1,2, \ldots, \\ \psi_{k}=\chi_{R_{+}}-\varphi_{k},\end{cases} \end{cases}
$$

$$
\begin{aligned}
\mathscr{H} f(x)= & 2^{-x} \int_{0}^{+\infty} \frac{2^{y} f(y)}{x-y} d y \\
= & \sum_{k \geq 0} 2^{-x} \varphi_{k}(x) \int_{k}^{k+1} \frac{2^{y} f(y)}{x-y} d y \\
& +\sum_{k \geq 0} 2^{-x} \int_{k}^{k+1}\left(\frac{\psi_{k}(x)}{x-y}-\frac{\psi_{k}([x])}{[x]-k}\right) 2^{y} f(y) d y \\
& +\sum_{k \geq 0} 2^{-x} \frac{\psi_{k}([x])}{[x]-k} \int_{k}^{k+1} 2^{y} f(y) d y \\
= & \mathscr{A} f(x)+\mathscr{B} f(x)+\mathscr{C} f(x) .
\end{aligned}
$$

Lemma A. The operator $\mathscr{A}$ is bounded on $L^{p, q}\left(\mathbb{R}_{+}, 4^{x} d x\right), 1<p<+\infty$ and $0<q \leq+\infty$.

Proof. Since the supports of the $\left\{\varphi_{k}\right\}$ are essentially disjoint, if $1<p<+\infty$,

$$
\begin{aligned}
\int_{0}^{+\infty}|\mathscr{A} f(x)|^{p} 4^{x} d x & \leq c \sum_{k \geq 0} \int_{k-1}^{k+2} 2^{(2-p) x}\left|\int_{k}^{k+1} \frac{2^{y} f(y)}{x-y} d y\right|^{p} d x \\
& \leq c \sum_{k \geq 0} 2^{(2-p) k} \int_{0}^{+\infty}\left|\int_{0}^{+\infty} \frac{\chi_{[k, k+1)}(y) 2^{y} f(y)}{x-y} d y\right|^{p} d x \\
& \leq c \sum_{k \geq 0} 2^{(2-p) k} \int_{0}^{+\infty}\left|\chi_{[k, k+1)}(x) 2^{x} f(x)\right|^{p} d x \\
& \leq c \int_{0}^{+\infty}|f(x)|^{p} 4^{x} d x .
\end{aligned}
$$

Hence $\mathscr{A}$ is bounded on $L^{p}\left(\mathbb{R}_{+}, 4^{x} d x\right)$. The boundedness on Lorentz spaces follows by interpolation.

Lemma B. The operator $\mathscr{B}$ is bounded on $L^{2, q}\left(\mathbb{R}_{+}, 4^{x} d x\right), 1 \leq q \leq+\infty$.

Proof. We observe that

$$
\begin{aligned}
|\mathscr{B} f(x)| & =\left|\sum_{k \geq 0} 2^{-x} \int_{k}^{k+1}\left(\frac{\psi_{k}(x)}{x-y}-\frac{\psi_{k}([x])}{[x]-k}\right) 2^{y} f(y) d y\right| \\
& \leq c 2^{-x} \sum_{k \geq 0} \frac{\int_{k}^{k+1} 2^{y}|f(y)| d y}{1+([x]-k)^{2}}=c T\left\{\beta_{j}\right\}(x),
\end{aligned}
$$


where $T$ has been defined in the previous section, and $\left\{\beta_{j}\right\}$ is the convolution (in $\mathbb{Z}$ ) of the sequences $\left\{\int_{k}^{k+1} 2^{y}|f(y)| d y\right\}$ and $\left\{1 /\left(1+k^{2}\right)\right\}$.

If the function $f$ belongs to $L^{2, q}\left(\mathbb{R}_{+}, 4^{x} d x\right), 1 \leq q \leq+\infty$, then by (iii) of the Proposition, the sequence $\left\{\int_{k}^{k+1} 2^{y}|f(y)| d y\right\}$ is in $l^{q}(\mathbb{N})$. Hence also the sequence $\left\{\beta_{j}\right\}$ is in $l^{q}(\mathbb{N})$, and the lemma follows from (i) of the Proposition.

Lemma $C$. The operator $\mathscr{C}$ is bounded on $L^{2, q}\left(\mathbb{R}_{+}, 4^{x} d x\right), 1<q<+\infty$, and from $L^{2,1}\left(\mathbb{R}_{+}, 4^{x} d x\right)$ into WEAK- $L^{2,1}\left(\mathbb{R}_{+}, 4^{x} d x\right)$.

Proof. As in the proof of the previous lemma we write

$$
\mathscr{C} f(x)=2^{-x} \sum_{k \geq 0} \psi_{k}([x]) \frac{\int_{k}^{k+1} 2^{y} f(y) d y}{[x]-k}=T\left\{\gamma_{j}\right\}(x),
$$

where $\left\{\gamma_{j}\right\}$ is the Hilbert transform (in $\mathbb{Z}$ ) of the sequence $\left\{\int_{k}^{k+1} 2^{y} f(y) d y\right\}$. A repeated use of the proposition completes the proof.

Parts (i) and (ii) of the theorem have been proven.

Proof of (iii). As in the proof of (i) and (ii) decompose

$$
\begin{aligned}
\mathscr{H} f(x) & =2^{-x} \int_{0}^{+\infty} \frac{2^{y} f(y)}{x-y} d y \\
& =\sum_{k \geq 0} 2^{-x} \varphi_{k}(x) \int_{k}^{k+1} \frac{2^{y} f(y)}{x-y} d y+\sum_{k \geq 0} 2^{-x} \psi_{k}(x) \int_{k}^{k+1} \frac{2^{y} f(y)}{x-y} d y \\
& =\mathscr{D} f(x)+\mathscr{E} f(x) .
\end{aligned}
$$

Lemma $D$. The operator $\mathscr{D}$ is bounded from $L^{1}\left(\mathbb{R}_{+}, 4^{x} d x\right)$ into WEAK$L^{1}\left(\mathbb{R}_{+}, 4^{x} d x\right)$.

Proof. Let $\mu$ denote the measure with density $4^{x} d x$ and $m$ the Lebesgue measure on $\mathbb{R}_{+}$. Then

$$
\begin{aligned}
\mu\{x & \left.\leq \mathbb{R}_{+}:|\mathscr{D} f(x)|>t\right\} \\
& \leq \sum_{k \geq 0} \mu\left\{x \in[k-1, k+2):\left|\int_{k}^{k+1} \frac{2^{y} f(y)}{x-y} d y\right|>\frac{2^{x} t}{3}\right\} \\
& \leq c \sum_{k \geq 0} 4^{k} m\left\{x \in \mathbb{R}_{+}:\left|\int_{k}^{k+1} \frac{2^{y} f(y)}{x-y} d y\right|>\frac{2^{k} t}{6}\right\} \\
& \leq c \sum_{k \geq 0} 4^{k} \frac{\int_{k}^{k+1}\left|2^{y} f(y)\right| d y}{2^{k} t} \leq c \frac{\int_{0}^{+\infty}|f(y)| 4^{y} d y}{t} .
\end{aligned}
$$

Lemma $\mathbf{E}$. The operator $\mathscr{E}$ is bounded from $L^{1}\left(\mathbb{R}_{+}, 4^{x} d x\right)$ into WEAK$L^{2,1}\left(\mathbb{R}_{+}, 4^{x} d x\right)$.

Proof. Observe that

$$
|\mathscr{E} f(x)| \leq c \sum_{k \geq 0} \frac{2^{-x}}{1+|x-k|} \int_{k}^{k+1}|f(y)| 2^{y} d y,
$$


and that the functions $\left\{2^{-x} /(1+|x-k|)\right\}$ are in WEAK- $L^{2,1}\left(\mathbb{R}_{+}, 4^{x} d x\right)$ with norm bounded independently of $k=1,2, \ldots$. Also, if $0<q<1$,

$$
\begin{aligned}
\sum_{k \geq 0}\left|\int_{k}^{k+1}\right| f(y)\left|2^{y} d y\right|^{q} & \leq\left\{\sum_{k \geq 0} 2^{-k q /(1-q)}\right\}^{1-q}\left\{\sum_{k \geq 0} \int_{k}^{k+1}|f(y)| 4^{y} d y\right\}^{q} \\
& =\left\{1-2^{-k q /(1-q)}\right\}^{q-1}\left\{\int_{0}^{+\infty}|f(y)| 4^{y} d y\right\}^{q} .
\end{aligned}
$$

Hence, since WEAK- $L^{2,1}\left(\mathbb{R}_{+}, 4^{x} d x\right)$ is $q$-normed for every $0<q<1$, then if the function $f$ is in $L^{1}\left(\mathbb{R}_{+}, 4^{x} d x\right)$,

$$
\sum_{k \geq 0} 2^{-x} /(1+|x-k|) \int_{k}^{k+1}|f(y)| 2^{y} d y
$$

converges in WEAK- $L^{2,1}\left(\mathbb{R}_{+}, 4^{x} d x\right)$ and the lemma is proved.

The proof of the theorem is then complete.

\section{FINAL REMARK}

In the introduction we defined the partial sums operators of the Fourier expansions of radial functions on the three-dimensional hyperbolic space. In [6] we proved that for every function $f$ in

$$
L^{3 / 2,1}\left(\mathbb{R}_{+},(\operatorname{Sinh} 2 x)^{2} d x\right)+\bigcup_{q<\infty} L^{2, q}\left(\mathbb{R}_{+},(\operatorname{Sinh} 2 x)^{2} d x\right)
$$

the partial sums $\left\{S_{R} f\right\}$ converge almost everywhere to $f$. We proved this result using an equiconvergence argument with cosine Fourier expansions, and obtaining no information on the norm convergence of the partial sums $\left\{S_{R} f\right\}$. Indeed, combining the techniques in [4] with those in this paper, it is not difficult to prove the following.

Theorem. For suitable test functions let $\mathscr{S}$ be the maximal operator associated to the partial sums $\left\{S_{R}\right\}, \mathscr{S} f(x)=\operatorname{Sup}_{R>0}\left|S_{R} f(x)\right|$. Then:

(i) The operator $\mathscr{S}$ is bounded on the Lorentz spaces $L^{2, q}\left(\mathbb{R}_{+},(\operatorname{Sinh} 2 x)^{2} d x\right)$, $1<q<+\infty$.

(ii) The operator $\mathscr{S}$ is bounded from $L^{2,1}\left(\mathbb{R}_{+},(\operatorname{Sinh} 2 x)^{2} d x\right)$ into WEAK$L^{2,1}\left(\mathbb{R}_{+},(\operatorname{Sinh} 2 x)^{2} d x\right)$.

(iii) The operator $\mathscr{S}$ is bounded from $L^{3 / 2,1}\left(\mathbb{R}_{+},(\operatorname{Sinh} 2 x)^{2} d x\right)$ into $L^{3 / 2, \infty}\left(\mathbb{R}_{+},(\operatorname{Sinh} 2 x)^{2} d x\right)+$ WEAK- $L^{2,1}\left(\mathbb{R}_{+},(\operatorname{Sinh} 2 x)^{2} d x\right)$.

This result is sharp, and does not extend to nonradial functions. For related results, see [5].

\section{REFERENCES}

1. C. Berenstein and N. Kerzman, Sur la réitération dans les espaces de moyenne, C. R. Acad. Sci. Paris Sér. I Math. 263 (1966), 609-612.

2. J. Bergh and J. Löfström, Interpolation spaces, Springer-Verlag, New York and Berlin, 1976.

3. C. Bennett and R. Sharpley, Interpolation of operators, Academic Press, New York, 1988.

4. S. Chanillo, The multiplier for the ball and radial functions, J. Funct. Anal. 55 (1984), $18-24$. 
5. L. Colzani, The multiplier for the ball on Lorentz spaces, preprint.

6. L. Colzani, A. Crespi, G. Travaglini, and M. Vignati, Equiconvergence theorems for FourierBessel expansions with applications to the harmonic analysis of radial functions in euclidean and non euclidean spaces (to appear).

7. T. Koornwinder, Jacobi functions and analysis on noncompact semisimple Lie groups, in R. Askey et al.: Special functions: Group Theoretical Aspects and Applications, Reidel, Dordrecht, 1984, pp. 1-85.

Dipartimento di Matematica, Universita' degli Studi di Milano, Via C. Saldini 50, 20133, Milano, Italia 